www.jmscr.igmpublication.org

Impact Factor (SJIF): 6.379

Index Copernicus Value: 71.58

ISSN (e)-2347-176x ISSN (p) 2455-0450

crossrefDOI: https://dx.doi.org/10.18535/jmscr/v6i7.178

Journal Of Medical Science And Clinical Research

\title{
A Study of Assessment of Mitral valve area before and after percutaneous balloon mitral valvuloplasty using Mitral leaflet separation index in a tertiary care center
}

\author{
Authors \\ Dr Nambirajan Jayabalan ${ }^{1}$, Dr Ravishankar Govindarajulu*², Dr Swaminathan \\ Nagarajan $^{3}$, Dr Elamaran Chidambaram ${ }^{4}$, Dr Prathap kumar Gorijavaram ${ }^{5}$ \\ ${ }^{1,4,5}$ Senior Assistant Professor, Institute of Cardiology, Madras Medical College, Chennai \\ ${ }^{2}$ Professor of Cardiology, Institute of Cardiology, Madras Medical College, Chennai \\ ${ }^{3}$ Director and HOD, Institute of Cardiology, Madras Medical College, Chennai \\ *Corresponding Author \\ Dr Ravishankar Govindarajulu \\ Professor of Cardiology, Institute of Cardiology, Madras Medical College, Chennai, India
}

\begin{abstract}
Background: Rheumatic fever and rheumatic heart disease continue to be endemic and leads the major acquired heart disease in India and other developing countries. The incidence of rheumatic fever and rheumatic mitral stenosis is very high in our country, having an incidence of 25-45\% of the acquired heart disease, with a women to men ratio of 2:1. Percutaneous balloon mitral commissurotomy is the best mode of nonsurgical treatment offering better results in suitable valves. Mitral leaflet separation (MLS) index is one of the best method to assess the severity of mitral stenosis. The aim of this study is to assess the Mitral valve area before and after percutaneous balloon mitral valvuloplasty using Mitral leaflet separation index in a tertiary care center

Methods: A retrospective study was done in the Institute of Cardiology, Madras Medical College, Chennai, Tamil nadu. Fifty patients with rheumatic mitral stenosis who underwent Percutaneous balloon mitral commissurotomy were evaluated clinically and echocardiographically during and after the procedure. In all patients the Mitral valve stenosis severity was assessed using mitral valve area in $2 D$ echo by planimetry, pressure half time and mitral leaflet separation index. The mitral leaflet separation index (MLS) was estimated by measuring average of distance between the maximal separation of tip of mitral valve leaflets in end-diastole in parasternal long axis and four chamber echocardiographic views.

Results: In our study, the mean age of the population was $20.51 \pm 10.22$ years and included mostly female gender (83\%). The mitral valve area measured after Balloon valvotomy increased from $0.80 \pm 0.13 \mathrm{~cm}^{2}$ to $1.65 \pm 0.36 \mathrm{~cm}^{2}$ as measured by echocardiography. Pre procedure PTMC the mean MVA (PHT) was 0.80 $\pm 0.25 \mathrm{~cm}^{2}$ and MLS index was 5.75 to $6.25 \mathrm{~mm}$. and after the procedure (PTMC) the mean MVA (PHT) was $1.65 \pm 0.36 \mathrm{~cm}^{2}$ and Mitral leaflet separation index (MLSI) was 10.22 to $15.55 \mathrm{~mm}$. In our study we noted, better correlation between the measured mitral valve area by echocardiography and mitral leaflet separation index (MLSI) $(r=0.36, p=0.0132)$ and also between MVA (PHT) and mitral leaflet separation index $(M L S I)(r=0.54, p=0.0001)$.

Conclusion: By using simple non invasive, easily available echocardiography method, the mitral leaflet separation index (MLSI) we can assess the severity of mitral stenosis before and after Percutaneous balloon mitral commissurotomy (PTMC).
\end{abstract}




\section{Introduction}

In our country and in other developing nations, Rheumatic heart disease remains a major cardiovascular problem, resulting in high mortality and morbidity ${ }^{1}$. Isolated mitral stenosis occurs in $40 \%$ of these patients. The prevalence of rheumatic heart disease (RHD) has declined sharply in developed countries during the last decade it continues to be predominant acquired cardiovascular disease in children and adolescents in our country. RHD is responsible for more than $25 \%$ of all admissions in patients with heart failure in endemic countries. ${ }^{2,3}$ A recent study by Indian Council of Medical Research (ICMR) (between 2000 and 2010) in various locations of the country found the prevalence to range from 0.2 to $1.1 / 1000$ for RHD and 0.0007 to $0.2 / 1000$ for Rheumatic fever. The overall prevalence estimated to be about 1.5-2/1000 in all age groups in India. There are about 2.0 to 2.5 million patients of RHD in the country and the global burden of RHD in the 5 to 14 years old children was estimated to be $0.8-5.7 / 1000$ with a median of $1.3 / 1000 .^{4,5}$

Rheumatic fever causes permanent damage to the cardiac valves, the mitral, aortic, tricuspid and pulmonary valves are involved in order of frequency, Mitral valve is the commonest valve involved and the pulmonary valve involvement is rare. Because of disease affecting younger age group, with high morbidity and mortality, in adolescents and young adults, it results in major causes of loss of productive years of life in our country ${ }^{6,7}$. With the identification of subclinical carditis and inclusion of echo criteria in diagnosis of rheumatic heart disease, the total burden of RHD is much higher than that estimated in various studies ${ }^{8,9}$. Mitral stenosis (MS) is a progressive disease, leading to symptoms like palpitations, dyspnea, fatigue and include fatal complications like pulmonary hypertension, pulmonary edema, systemic embolism due to arrhythmias. $^{10,11}$

Management comprises of medical therapy to relieve symptoms, and finally if it does not to relieve the symptoms of valvular obstruction we need to dilate the valve either surgically or by ballon. The surgical commissurotomy both open and closed were the only methods for many years by which mitral stenosis could be corrected. But after the development of percutaneous balloon mitral valvuloplasty (PBMV) by Inoue in 1984 and Lock in 1985 for the treatment of mitral stenosis in suitable patients, there was a revolution in the treatment of mitral stenosis worldwide. ${ }^{12,13}$ Easy availability, less procedure time, the long term results, lower costs, and the avoidance of surgery make PBMV the treatment of choice in patients with mitral stenosis who have the suitable valve morphology echocardiographically ${ }^{14}$.

Patients with the following suitable criteria are selected for the procedure, patient with moderate to severe mitral stenosis, noncalcified, pliable mitral valves and patients with symptomatic or asymptomatic pulmonary arterial hypertension, most importantly the absence of left atrial thrombus and absence of moderate to severe mitral regurgitation.

PBMV done as a palliative procedure in patients who are too sick, older age and not suitable for surgical intervention. In all patients with mitral stenosis at initial presentation echocardiography evaluation is done and yields more accurate diagnosis and is recommended periodically at regular intervals for monitoring disease progression. In certain obese individuals, COPD patients if the transthoracic echo views are suboptimal, transesophageal echocardiography (TEE) is recommended. and also a must to exclude left atrial thrombus and to evaluate mitral regurgitation severity. In our study all patients with mitral stenosis were subjected to a detailed echocardiographic examination, including 2D echocardiography (transthoracic or transesophageal), color flow doppler imaging.

The mitral leaflet separation index is the distance measured between the tips of the mitral leaflets in end diastole in parasternal long-axis and fourchamber views ,these two readings are averaged to give the final mitral leaflet separation index. In 
recent studies it was presented as a reliable measure of MS severity and a suitable index for mitral valve area (MVA).

\section{Objective}

The aim of this study is to assess the mitral valve area measured by the mitral leaflet separation index (MLSI) index and with 2D echocardiography parameters such as planimetry method and Doppler PHT method in patients before and after PTMC.

\section{Methods}

This study was a retrospective observational study which included all patients undergoing elective PTMC in the Institute of Cardiology, Madras Medical College, Chennai, Tamilnadu. Fifty patients with rheumatic mitral stenosis who underwent Percutaneous balloon mitral commissurotomy (PTMC) were included in this study and informed consent was obtained from all individuals enrolled in the study. Instituitional ethical commitie approval was obtained.

\section{Study population}

The patients with following criteria were included in our study, patients aged from 18 to 50 years, Symptomatic moderate to severe mitral stenosis, patients with moderate to severe pulmonary hypertension, patients in sinus rhythm or atrial fibrillation.

The following criteria was used for exclusion, patients with mild MS (MVA > $1.5-2.0 \mathrm{~cm} 2$ ), patients not suitable for PTMC (due to either Wilkins score > 10, patients with commissural calcification or left atrial thrombus), associated other severe valvular disease other than MS, evidence of rheumatic activity during the preceding 6 months, congenital mitral stenosis, and in patients with organic tricuspid valve disease.

\section{Evaluation by trans-thoracic echocardiography}

In this study all patients were subjected to detail 2D echo using an echocardiography machine (PHILIPS AND ALOKA). Standard 2D echo views with M-mode echocardiograms were done according to the American Society of Echocardiography guidelines. The basic routine indices for assessing the severity of mitral stenosis including MVA by planimetry, the pressure halftime and mean mitral valve pressure gradients and pulmonary artery systolic pressure were recorded. In our study we defined severe mitral stenosis as a MVA of $1 \mathrm{~cm}^{2}$ or less by planimetry or pressure half-time method and/or a mean trans mitral gradient of greater than $10 \mathrm{mmHg}$, moderate mitral stenosis was defined as a MVA between 1 $\mathrm{cm}^{2}$ and $1.5 \mathrm{~cm}^{2}$ by planimetry or pressure halftime method, with a mean transmitral gradient of 5 to $10 \mathrm{~mm} \mathrm{Hg}$ and mild mitral stenosis was defined as a MVA of greater than $1.5 \mathrm{~cm}^{2}$ by planimetry or pressure half-time and/or a trans mitral gradient of less than $5 \mathrm{~mm} \mathrm{Hg}{ }^{15,16}$

In assessing suitability of mitral valve we used Wilkins scoring system of mitral valve on scale of 1 through 4, with score of 1 representing normal, we studied the four parameters including the mobility of the anterior leaflet, the severity of subvalvular disease, the calcification of the anterior leaflet, and the thickness of the anterior leaflet. The total score for each of these four indices was added for a total of $16 .^{17}$

In our study severe mitral regurgitation was defined as a large central color doppler jet area more than $10 \mathrm{~cm}^{2}$ or more than $50 \%$ of left atrium area or variable size wall-impinging jet whole of left atrium, moderate mitral regurgitation was defined as a color doppler jet area greater than mild but no criteria for severe MR or $15-30 \%$ of LA. Mild mitral regurgitation was defined as small, central jet less than $4 \mathrm{~cm}^{2}$ or less than $15 \%$ of left atrium area. ${ }^{18}$ In this study the Mitral Leaflets Separation (MLS) index was defined as the maximal separation of the mitral valve leaflet tips which was measured from inner edge to inner edge in end diastole both in the para sterna long axis and in apical 4-chamber views. These two readings were averaged to determine the final MLS index. 
Percutaneous balloon mitral commissurotomy (PTMC)

\section{Procedure}

- Right femoral artery and femoral vein accessed with $6 \mathrm{~F} \& 7 \mathrm{~F}$ hemostatic sheaths respectively. LV angio done with $10 \mathrm{ml}$ contrast by hand injection in RAO $30 \mathrm{deg}$ using $6 \mathrm{~F}$ pigtail catheter.

- 0.032" wire was placed in left innominate vein. IAS punctured with 8 F Mullin's sheath and Brocken borough needle in lateral position by Modified Hung's technique. LA pressure obtained.

- 0.025 " coil wire placed in LA and septal puncture dilated using $14 \mathrm{~F}$ plastic dilator

- Mitral valve crossed with help of stylet in RAO 30 position \& dilatation given using $24 \mathrm{~mm}$ ACCURA balloon for $26 \mathrm{~mm}$ size twice.

- Mitral valve area measured 1.4 sq.cm by planimetry by echo and both commissures appear split.

- Sheaths removed. Hemostasis secured and patient shifted to ICU for monitoring.

\section{Statistical analysis}

The data were analysis using SPSS (Statistical Package for Social Science) Ver 16.01. The pair sample t-test was used for testing the significance of the pre and post, mean \& standard deviation. $\mathrm{P}$ value $\leq 0.05$ was considered as statistically Significant in all the statistical results.

\section{Results}

In our study, the mean age of the population was $20.51 \pm 10.22$ years and included mostly female gender $(83 \%)$. In our study $70 \%$ of the patients suffered severe mitral stenosis (MS) and the remaining 30\% suffered moderate mitral stenosis as assessed by $2 \mathrm{D}$ planimetry echocardiography. The mitral valve area measured after the procedure PTMC, it increased from $0.80 \pm 0.13$ $\mathrm{cm} 2$ to $1.65 \pm 0.36 \mathrm{~cm} 2$ as measured by echocardiography. Pre procedure PTMC the mean
MVA (PHT) was $0.77 \pm 0.25 \mathrm{~cm} 2$ and MLS index was $5.75 \pm 1.25 \mathrm{~mm}$. and after the procedure (PTMC) the mean MVA (PHT) was $1.73 \pm 0.42$ $\mathrm{cm} 2$ and Mitral leaflet separation index (MLSI) was $10.22 \pm 4.24 \mathrm{~mm}$. In most of patients Successful PTMC was achieved with significant increase in planimetry and pressure half time (PHT) measured MVA as well as significant drop in mean gradient across the mitral valve and pulmonary artery systolic pressure (Table 1) where $90 \%$ of the study Population improved to mild MS while $10 \%$ improved to moderate MS Before BMV 32 patients (76.7\%) had no MR and 18 patients $(23.3 \%)$ had mild MR. Post PBMV 18 patients $(27.8 \%)$ had no MR, 12 patients $(25.6 \%)$ had mild MR, 16patients (31.1\%) had moderate MR and 4 patients $(6.7 \%)$ had severe MR 2 of them $(3.3 \%)$ transferred to surgery. In our study all patients data were recorded and distributed according MVA using, both 2D planimetry and PHT methods were generated to determine the cut off values of MLS index for both methods of MVA measurements. In our study we noted, better correlation between the measured mitral valve area by echocardiography and mitral leaflet separation index (MLSI) $\mathrm{p}=0.019$ ) and also between MVA (PHT) and mitral leaflet separation index (MLSI) $(\mathrm{p}=0.001)$. 


\section{ECHO Parameters of Mitral Stenosis before and after PTMC}

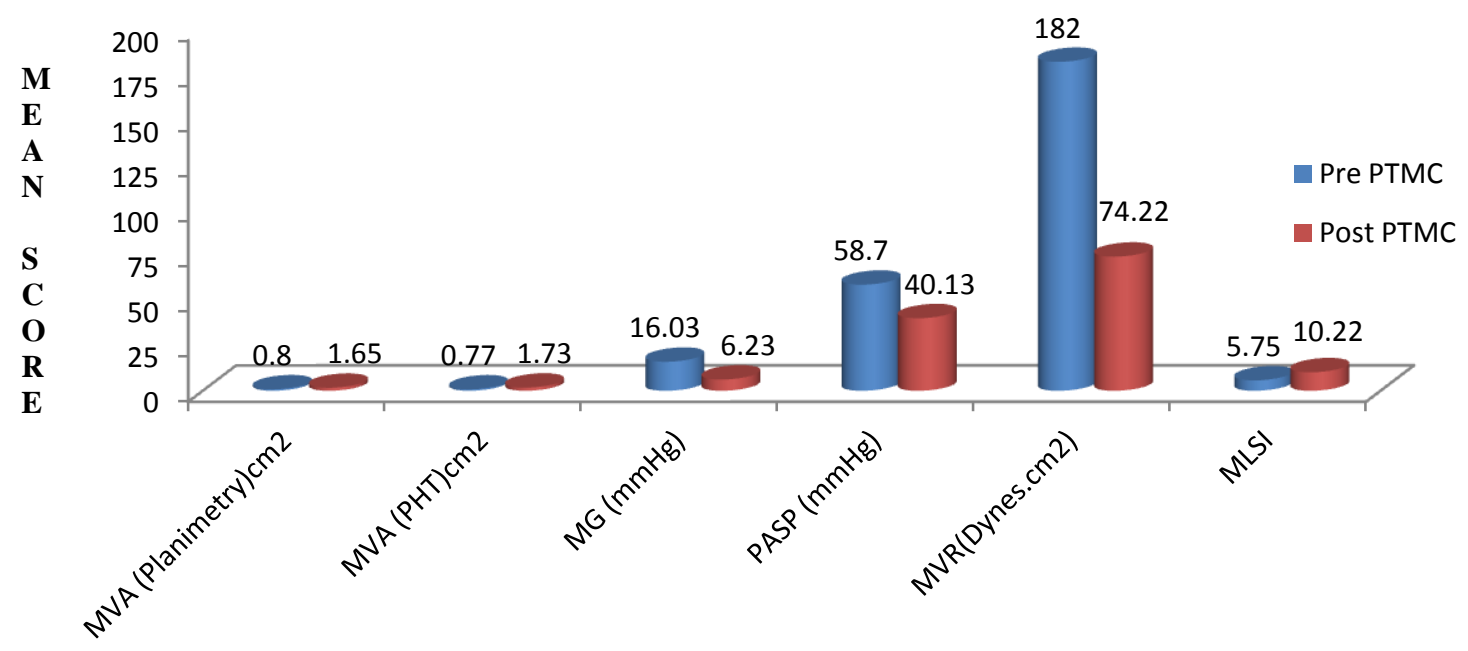

Table-1 Baseline characteristics of study subjects before PTMC

\begin{tabular}{|l|c|c|c|c|}
\hline Variables & Minimum & Maximum & Mean & Standard deviation \\
\hline Age & 20 & 50 & 35.67 & 10.02 \\
\hline MVA (Planimetry) in $\mathrm{cm}^{2}$ & 0.73 & 1.50 & 1.05 & 0.22 \\
\hline MVA (PHT) in $\mathrm{cm}^{2}$ & 0.75 & 1.63 & 1.08 & 0.32 \\
\hline Mean Gradient (mmHg) & 10.25 & 22.02 & 16.03 & 4.35 \\
\hline
\end{tabular}

Table-2 ECHO parameters of mitral stenosis before and after PTMC

\begin{tabular}{|l|c|c|c|}
\hline Variables & Pre PTMC & Post PTMC & p-value \\
\hline MVA (Planimetry) in $\mathrm{cm}^{2}$ & $0.80 \pm 0.13$ & $1.65 \pm 0.36$ & 0.19 \\
\hline MVA (PHT) in $\mathrm{cm}^{2}$ & $0.77 \pm 0.25$ & $1.73 \pm 0.42$ & 0.001 \\
\hline MGin $\mathrm{mmHg}$ & $16.03 \pm 4.36$ & $6.23 \pm 1.25$ & 0.001 \\
\hline PASP $(\mathrm{mmHg})$ & $58.70 \pm 0.38$ & $40.13 \pm 4.37$ & 0.002 \\
\hline MVR(Dynes. $\left.\mathrm{cm}^{2}\right)$ & $182.00 \pm 4.34$ & $74.22 \pm 5.62$ & 0.002 \\
\hline MLSI & $5.75 \pm 1.25$ & $10.22 \pm 4.24$ & 0.001 \\
\hline
\end{tabular}

\section{Discussion}

Rheumatic fever and Rheumatic heart disease is still the leading cause for cardio vascular morbidity and mortality in India. In India rheumatic fever is endemic and remains one of the major causes of cardiovascular disease accounting for nearly $25-45 \%$ of the acquired heart disease. In recently conducted study it was found that the annual incidence of rheumatic fever is 100-200 times greater than that observed in developed countries and fluctuates between 100-200 per $1,00,000$ children of school age.

The treatment of mitral stenosis consists of medical management to alleviate symptoms to some extent and finally for surgical management either open orclosed mitral commisurotomy apart from mitral valve replacement. The concept of mitral commisurotomy was first proposed by Brunton in 1902 and the first successful closed mitral commisurotomy was done in 1923 by Elliot 
Cutler. Later on in 1976 Kanji Inoue $^{13}$, a cardiovascular surgeon from Japan developed the new ballon catheter system, which was finally shaped up in 1980, and in 1982 onwards the inoue ballon was in clinical use and several modifications of the BMV technique have been introduced over the years.

Many studies have established the safety and efficacy of PTMC ${ }^{20}$ and PTMC is the treatment of choice in patients with suitable anatomy.

Echocardiography evaluation of mitral stenosis is more accurate for diagnosis. By 2-D echo we can measure the mitral valve area (MVA) by planimetry, pressure half-time, continuity equation, and proximal isovelocity surface area methods. and the MVA measured by 2D Planimetry is considered as the reference method but must be done precisely at the tips of the leaflets. Trans mitral gradient and the continuity equation depend upon transvalvular flow and are easily affected by cardiac output and presence of mitral regurgitation ${ }^{.21,22}$

Using mitral leaflet separation (MLS) index as a measure of MS severity was first proposed by Fisher et al. in 1979. and measured the maximum diastolic separation of the mitral valve leaflets by M-mode echocardiography and found a good correlation with MVA obtained invasively using Gorlin formula ${ }^{23}$

The measurement of MLS on 2D imaging is technically easier and more accurate

The MLS demonstrated a very good correlation with MVA measured with 2D planimetry and PHT methods and is very easier to measure and correlate and a simple and effective method for assessment of the MVA

In our study 50 patients with mitral stenosis of varying severity were evaluated before and after PBMV using 2D planimetry and PHT for obtaining MVA by measuring MLS index. 42 patients were females $(77.78 \%)$ and 8 of them were males $(22.22 \%){ }^{24,25}$

Our study demonstrated that percutaneous balloon mitral valvuloplasty (PBMV) is a successful method to improve the mitral valve area and mean pressure gradient across the valve. ${ }^{26,27}$ The mitral valve area measured after the procedure PTMC, it increased from $0.80 \pm 0.13 \mathrm{~cm} 2$ to $1.65 \pm 0.36 \mathrm{~cm} 2$ as measured by echocardiography. Pre procedure PTMC the mean MVA (PHT) was $0.77 \pm 0.25 \mathrm{~cm} 2$ and MLS index was $5.75 \pm 1.25 \mathrm{~mm}$. and after the procedure (PTMC) the mean MVA (PHT) was $1.73 \pm 0.42 \mathrm{~cm} 2$ and Mitral leaflet separation index (MLSI) was $10.22 \pm 4.24 \mathrm{~mm}$, our study results are comparable with the results from other studies conducted all over world ${ }^{28,29 .}$

\section{Conclusion}

By using simple easily available, non invasive echocardiography method, the Mitral leaflet separation index (MLSI) we can assess the severity of mitral stenosis before and after Percutaneous balloon mitral commissurotomy (PTMC). When compared to planimetry, the MLS index is technically much easier to measure especially in patients with poor echo window. MLS index is a quick estimate of MS severity from standard 2D echocardiographic views without much difficulty especially in the setting of PTMC during procedure in the cath lab, the MLS index can be used as complimentary and cannot be substituted for standard echo parameters such as planimetry, pressure half time and mean gradient in assessing the mitral valve area

\section{References}

1. Padmavathi S. Present status of Rheumatic fever and rheumatic heart disease in India. Indian Heart Journal 1995;47: 395-398.

2. Padmavathi S. Rheumatic fever and rheumatic heart disease in India at the turn of the Century. Indian Heart Journal 2002; 53: 35-37.

3. WHO. Rheumatic fever and rheumatic heart disease: report of a WHO Expert Consultation, Geneva 29 October - 1 November 2001. WHO technical report series; 923.Geneva: World Health Organisation, 2004. 
4. WHO Technical Report, Series. Rheumatic fever and rheumatic heart disease: 1 November 2001. Geneva: WHO; 200

5. IB Vijayalakshmi. Rheumatic Fever, Rheumatic Heart Disease Registry and Control Program. IB Vijayalakshmi ed. Acute Rheumatic Fever and Chronic Rheumatic Heart disease. 2011. Jaypee Brothers Medical Publishers. India. P324336.

6. Mishra TK, Acute Rheumatic Fever and Rheumatic Heart Disease: Current Scenario. Indian Academy of Clinical Medicine 2007;8:324-30.

7. Ntusi NB, Mayosi BM. Epidemiology of heart failure in sub-Saharan Africa. Expert Rev Cardiovasc Ther. 2009;7:169.

8. Vijayalakshmi IB, Mithravinda J, Deva AN. The role of echocardiography in diagnosing carditis in the setting of acute rheumatic fever. Cardiol Young 2005; 15: 583-588

9. Bocchi EA, Guimaraes G, Tarasoutshi F, et al.. Cardiomyopathy: adult valve disease and heart failure in South America. Heart. 2009;95:181.

10. Olson LJ, Subramanian R, Ackermann DM, et al.. Surgical pathology of the mitral valve: a study of 712 cases spanning 21 years. Mayo Clin Proc. 1987;62:22.

11. Horstkotte D, Niehues R, Strauer BE. Pathomorphological aspects: aetiology and natural history of acquired mitral valve stenosis. Eur Heart J. 1991;12:55.

12. Carroll JD, Feldman T. Percutaneous mitral balloon valvotomy and the new demographics of mitral stenosis. JAMA. 1993;270:1731.

13. Inoue $\mathrm{K}$, Owaki T, Nakamura T, et al.. Clinical application of transvenous mitral commissurotomy by a new balloon catheter. J Thorac Cardiovasc Surg. 1984;87:394.
14. Lock JE, Khalilullah M, Shrivastava S, et al.. Percutaneous catheter commissurotomy in rheumatic mitral stenosis. N Engl J Med. 1985;313:1515.

15. Otto CM. Valvular heart disease: mitral stenosis. Philadelphia Saunders. Vol.252, 2nd ed. 2004. p. 255.

16. Bonow RO, Carabello BA, Chatterjee K, et al.. ACC/AHA 2006 guidelines for the management of patients with valvular heart disease: A report of the American College of Cardiology/American Heart Association Task Force on Practice Guidelines (writing committee to revise the 1998 Guidelines for the Management of Patients with Valvular Heart Disease): Developed in collaboration with the Society of Cardiovascular Anesthesiologists: endorsed by the Society for Cardiovascular Angiography and Interventions and the Society of Thoracic Surgeons. Circulation. 2006;114:184.

17. Inoue K, Feldman T. Percutaneous transvenous mitral commissurotomy using Inoue balloon catheter. Cathet Cardiovasc Diagn. 1993;28:119.

18. Wilkins GT, Weyman AE, Abascal VM, et al.. Percutaneous balloon dilatation of the mitral valve: an analysis of echocardiographic variables related to outcome and the mechanism of dilatation. Br Heart J. 1988;60:299.

19. Feigenbaum H, Armstrong WF, Ryan T, et al. Feigenbaum's Echocardiography. Mitral valve disease. Lippincott Williams \& Wilkins. Vol. 11, 6th ed.; 2005. p.30

20. Vahanian A, Baumgartner H, Bax J, et al.. Guidelines on the management of valvular heart disease: the Task Force on the Management of Valvular Heart Disease of the European Society of Cardiology. Eur Heart J. 2007;28:230.

21. Messika-Zeitoun D, Lung B, Brochet E, Himbert D, et al. Evaluation of mitral 
stenosis. Arch Cardiovasc Dis. 2008; 653:63.

22. Rifaie O, Abdel-Dayem MK, Ramzy A, Ezz-El-Din H, et al. Percutaneous mitral valvotomy versus closed surgical commissurotomy: up to 15 years of follow-up of a prospective randomized study. J Cardiol. 2009; 53:28-34.

23. Faletra F, Pezzano Jr A, Fusco R, Mantero A, et al.. Measurement of mitral valve area in mitral stenosis: four echocardiographic methods compared with direct measurement of anatomic orifices. J Am Coll Cardiol. 1996;28:1190.

24. Seow SC, Koh LP, Yeo TC. Hemodynamic significance of mitral stenosis: use of a simple, novel index by 2dimensional echocardiography. J Am Soc Echocardiogr. 2006;19:102-106.

25. Fisher ML, Parisi AF, Plotnick GD, DeFelice CE, et al.. Assessment of severity of mitral stenosis by echocardiographic leaflet separation-. Intern Med. 1979; 139:402.

26. Holmin C, Messika-Zeitoun D, Mezalek AT, Brochet E, et al.. Mitral leaflet separation index: a new method for the evaluation of the severity of mitral stenosis? Usefulness before and after percutaneous mitral commissurotomy. J Am Soc Echocardiogr. 2007;1119:24.

27. Duggal B, Bajaj M, Prabhu S, Mathew M. The mitral leaflet separation index for assessment mitral stenosis during percutaneous mitral commissurotomy: validation of the index in the immediate post PMC period. Echocardiography. 2012;9:10

28. Fawzy ME, Shoukri M, Al Buraiki J, Hassan W, et al.. Seventeen years' clinical and echocardiographic follow up of mitral balloon valvuloplasty in 520 patients, and predictors of long-term outcome. J Heart Valve Dis. 2007;16 (454):60.
29. Boscarini M, Repetto S, Secchin G, Stifani A, et al.. Percutaneous mitral commissurotomy: immediate and short-term result. G Ital Cardiol. 1991;21 (1185):94. 